\title{
Mitosis of Hepatitis B virus-infected cells results in uninfected daughter cells
}

\section{Thomas Tu ( $\nabla$ t.tu@sydney.edu.au )}

Westmead Institute for Medical Research

\section{Benno Zehnder}

University Hospital Heidelberg https://orcid.org/0000-0002-4910-2636

\section{Jochen Wettengel}

Institute of Virology, School of Medicine, Technical University of Munich / Helmholtz Zentrum München

\section{Henrik Zhang}

The Westmead Institute for Medical Research

\section{Sally Coulter}

University of Sydney

\section{Vikki Ho}

Storr Liver Centre

\section{Mark Douglas}

https://orcid.org/0000-0003-4621-3485

\section{Ulrike Protzer}

School of Medicine, Technical University of Munich / Helmholtz Zentrum München https://orcid.org/0000-0002-9421-1911

\section{Jacob George}

The Westmead Institute for Medical Research https://orcid.org/0000-0002-8421-5476

\section{Stephan Urban}

University Hospital Heidelberg

\section{Article}

Keywords: Covalently closed circular DNA, Hepatitis B virus, viral persistence, cinqPCR

Posted Date: February 9th, 2022

DOI: https://doi.org/10.21203/rs.3.rs-1325894/v1

License: (c) (i) This work is licensed under a Creative Commons Attribution 4.0 International License. Read Full License 
1 Title: Mitosis of Hepatitis B virus-infected cells results in uninfected daughter cells

2

3 Authors:

4 Thomas $\mathrm{Tu}^{1,2}$, Benno Zehnder ${ }^{3}$, Jochen M. Wettengel ${ }^{4}$, Henrik Zhang ${ }^{1}$, Sally Coulter ${ }^{1}$, Vikki Ho ${ }^{1}$, Mark

5 W. Douglas ${ }^{1,2}$, Ulrike Protzer ${ }^{4,5}$, Jacob George ${ }^{1 *}$, Stephan Urban ${ }^{3,6 *}$

6

7 Affiliations:

$8 \quad{ }^{1}$ Storr Liver Centre, Westmead Clinical School and Westmead Institute for Medical Research, Faculty

9 of Medicine and Health, The University of Sydney, Westmead, NSW 2145, Australia

$10 \quad{ }^{2}$ Sydney Institute for Infectious Diseases, University of Sydney at Westmead Hospital, Westmead

11 NSW 2145, Australia.

$12{ }^{3}$ Department of Infectious Diseases, Molecular Virology, University Hospital Heidelberg, Heidelberg,

13 Germany

$14{ }^{4}$ Institute of Virology, Technische Universität München/Helmholtz Zentrum München, Munich,

15 Germany.

$16{ }^{5}$ German Center for Infection Research (DZIF), Munich Partner Site, Munich, Germany

$17{ }^{6}$ German Center for Infection Research (DZIF), Heidelberg Partner Site, Heidelberg, Germany

18

19 Corresponding Author: Dr. Thomas Tu, t.tu@sydney.edu.au; thomas.tu.phd@gmail.com

20 Keywords: Covalently closed circular DNA, Hepatitis B virus, viral persistence, cinqPCR 
24 Chronic hepatitis B is a major cause of liver failure and liver cancer, resulting in 600,000 annual

25 deaths globally. Infection chronicity (and resultant liver disease) is determined by intrahepatic

26 persistence of the viral covalently closed circular DNA (cccDNA), an episomal form that encodes all

27 viral transcripts. Therefore, cccDNA is a key target for new treatments, with the ultimate aim of its

28 elimination. While established cccDNA molecules are stable in resting hepatocytes, their fate in

29 dividing cells is not well understood. Using highly-sensitive quantification assays, we observed a

30 dramatic reduction in cccDNA levels, HBV-positive cell numbers, and cccDNA-dependent protein

31 expression after each round of cell mitosis. These observations are consistent with a complete loss

32 (as opposed to a dilution) of cccDNA in daughter cells. Our results thus show that HBV persistence

33 can be efficiently overcome by inducing cell mitosis and justify therapeutic approaches that induce

34 liver turnover (e.g. immune modulators) as an adjunct to achieve Hepatitis B cure. 
Hepatitis B virus (HBV) is an enveloped, partially double-stranded DNA virus and the prototypic member of the Hepadnaviridae. Chronic infection with HBV confers a high risk of developing liver cirrhosis and hepatocellular carcinoma, causing 600 000 deaths annually ${ }^{1}$. HBV is not cytopathic; instead, liver injury is driven by virus-induced inflammatory immune responses that are insufficient to eradicate infected hepatocytes. Thus, chronic HBV infection is often a life-long and incurable condition, which in turn drives other personal impacts (e.g. anxiety about disease progression, stigma and discrimination, and long-term health care costs) ${ }^{2}$.

Persistence of covalently closed circular viral DNA (cccDNA) in the hepatocyte is the critical determinant of chronic hepatitis B infection. cccDNA is an episomal plasmid-like "mini-chromosome" that acts as the transcriptional template for HBV mRNAs and the pregenomic RNA and is therefore necessary for viral replication. However, the host immune responses are usually sub-optimal and cannot fully eliminate all cccDNA-containing hepatocytes. Given its supercoiled structure and complexing with cellular histones, cccDNA is stable within an infected hepatocyte (likely for its entire lifetime) ${ }^{3,4}$, though some studies have suggested partial non-cytolytic clearance ${ }^{5-7}$. Additional routes of cccDNA elimination from the liver are immune-mediated killing of cccDNA-containing hepatocytes and possibly loss following mitosis ${ }^{8}$. Understanding the mechanisms behind the elimination of cccDNA will greatly aid in developing therapeutics to overcome chronic HBV infections.

Elimination of cccDNA in infected hepatocytes would result in a cure of chronic $\mathrm{HBV}^{9}$, which cannot be efficiently achieved by current treatment options. Clearance of cccDNA would allow cessation of therapy without viral rebound, thereby slowing liver pathogenesis. Mathematical models predict that various independent parameters (including the cccDNA copy number per cell, its rate of degradation, and its stability in resting and replicating cells) profoundly affect the dynamics of cccDNA clearance ${ }^{10,11}$. These dynamics are not fully resolved as there is controversy as to whether 
61 mitosis of an infected cell results in two uninfected daughter cells $s^{8,12}$ or if cccDNA survives and is

62 diluted amongst the daughter cells $s^{4,10,13,14}$.

64 The reduction of cccDNA levels with mitosis critically determines the optimal cure strategy: for

65 example, if the cccDNA pool of an infected hepatocyte is lost within one round of mitosis, then

66 activation of antiviral immunity or other methods to induce turnover of infected cells become an

67 attractive option for treatment. On the other hand, if cccDNA survives mitosis greater emphasis on ccCDNA degrading pathways (e.g. CRISPR-Cas9) or direct killing of infected cells (e.g. CAR-T cell technology) may be of higher priority. Given the central importance of cccDNA reduction for how the field should proceed with therapeutic cures of chronic hepatitis B, we aimed to clarify the fate of cccDNA after cell mitosis.

73 We used a novel $\underline{c} c$ DNA inversion quantitative (cinq)PCR assay ${ }^{15,16}$ to precisely quantify cccDNA

74 levels. We find that cccDNA levels undergo a $~ 5$-fold decrease after each round of mitosis, which is

75 the exact rate predicted by mathematical models assuming a complete loss of cccDNA in daughter

76 cells. Evidence from orthogonal approaches using reporter HBV viruses was consistent with this

77 model, down to the level of a single cell. Thus, we unequivocally show that liver turnover is an

78 efficient mechanism to clear HBV cccDNA, which informs future therapeutic approaches to the cure

79 of chronic hepatitis B. 
We predicted the levels of cccDNA following cell mitosis given each of the two major hypothetical models: i) the cccDNA loss model, where the cccDNA pool in an infected cell is not conveyed at all to the daughter cells after mitosis; and ii) the cccDNA dilution model, where the entire cccDNA pool is distributed amongst the daughter cells.

We assumed that each cell has an equal probability of undergoing mitosis, thereby resulting in a Poisson frequency distribution in rounds of mitosis per cell. This assumption predicts that $63.2 \%$ of cells would undergo at least one mitosis cycle following an average of one cell division of the bulk population. Thus, in the model where cccDNA is completely lost after mitosis, $36.8 \%$ of cells maintain their cccDNA. Assuming cccDNA is diluted upon mitosis and no new cccDNA is formed ${ }^{16}$, the cccDNA copy numbers do not change.

93

In both the loss and dilution models, the cell population doubles; thus the number of cccDNA molecules per cell reduces by $50 \%$. Thus, the loss model predicts an average net reduction to $18.4 \%$ of initial cccDNA levels per cell after each round of mitosis, whereas the dilution model predicts a reduction to $50 \%$ per round of mitosis. In both models, this predicted reduction is independent of the size of the cccDNA pool per individual cell. in daughter cells We tested these predictions in two different HBV-susceptible cell lines, HepG2-NTCP and HepaRGNTCP cells. After initial cccDNA establishment (3 days post-infection, dpi), the infected cells were serially passaged (1:2) every 3 days to induce cell mitosis. We developed two experimental setups 
(Figure 1A): Setup 1, where total cellular DNA was extracted immediately following the splitting procedure; and Setup 2, where cells were cultured until 12 dpi to control for any cccDNA level changes occurring during the time course (e.g. via cccDNA amplification or degradation in resting cells). cccDNA molecules per cell were then precisely quantified by cinqPCR. Decreases in cccDNA levels following splitting closely matched those expected from the loss model in both HepG2-NTCP (Figure 1B) and HepaRG-NTCP (Figure 1C) cells.

In the previous experiment, we had not blocked viral replication. We tested if the mitosis-dependent reduction in cccDNA levels could be accelerated by inhibiting viral replication, as cccDNA has been assumed to be replenished by nuclear import of mature nucleocapsids in the cytosol. Such a mechanism can be impeded using a replication-deficient virus. Therefore, we investigated the postmitotic levels of cccDNA in HepG2-NTCP cells infected with replication-deficient HBV with an early stop codon in the HBV core protein $(\mathrm{HBC})$ open reading frame ${ }^{16}$. If the nuclear import pathway was indeed active, then cccDNA levels would be lower in cells infected with HBc-deficient mutants compared to wild-type HBV. This experimental setup also tests the contribution of de novo HBV spread in the culture to cccDNA levels (though this is not expected to occur in cell culture as shown previously $\left.{ }^{16}\right)$.

We observed no difference in the rate of cccDNA reduction during serial rounds of mitosis between cells infected with HBc-deficient HBV or wild-type virus in Setups 1 or 2, as described in Figure 1 (Figure 2). We conclude that HBV spread, replication, or cytoplasmic mature nucleocapsids do not play significant roles in cccDNA levels after mitosis of HBV-infected daughter cells. Moreover, this information allows us to use replication-defective reporter virus to further investigate cccDNA loss, knowing that the lack of virus replication would not impact these dynamics. 
132 We determined if protein expression from cccDNA also declined at the same rate as cccDNA copy number. We found that the detection of secreted viral antigens (HBeAg and HBsAg) by ELISA was insufficiently sensitive to precisely and accurately determine the relatively low levels of secretion after several rounds of mitosis (data not shown). We instead used a reporter HBV encoding secreted Gaussia Luciferase under a transcriptionally-strong TTR-promoter (rHBV-TTR-GLuc) in place of the HBsAg open reading frame (ORF) to infect HepG2-NTCP cells and used a serial-splitting experimental design similar to setup 2 described above (Figure 3A). Luciferase activity can be detected with much higher sensitivity compared to HBV antigens and was measured in the supernatant of infected cells 6 days following passage (thereby allowing cells to divide and then secrete sufficient luciferase into the supernatant).

Reduction of luciferase activity paralleled the reduction of cccDNA levels expected by the loss model (i.e. a reduction of $\sim 5$-fold with each successive split) compared to the dilution model (2-fold reduction per split), except for cells after the third passage where high variation (due to low signal) prevented clear observation of signal reduction. Luciferase activity was relatively stable after reaching a plateau, suggesting there were no fluctuations due to temporary epigenetic changes of transgene expression after cellular mitosis. Luciferase activity of negative controls pre-treated with virus entry inhibitor Myrcludex B never exceeded $0.8 \%$ at any time point, indicating that all detected signals were due to bona fide HBV infection. In conclusion, we show that the HBV cccDNA that is lost with mitosis is transcriptionally-active and its loss parallels the reduction in viral protein expression. for HBc. HepG2-NTCPs showed a marked reduction in HBc-positive cell numbers after cell mitosis 
157 (Figure 4A). The HBc-positive cells after mitosis induction present as individual cells (likely infected

158 cells that have not undergone mitosis), which is consistent with the loss model. On the contrary, the

159 dilution model would predict some cluster formation of two or more adjacent HBc-positive cells.

160 Identical patterns were seen in HepaRG-NTCP cells (Figure S1). The number of HBc-positive cells

161 (Figure 4B) at each passage more closely matched mathematical models where $2 \mathrm{HBc}$-negative

162 daughter cells result from mitosis (complete loss), compared to those where either 1 or $2 \mathrm{HBC}$ -

163 positive daughter cells results from mitosis of infected cells (dilution model). Together, these data

164 provide further support for the complete loss model.

165

Live cell imaging showed daughter cells of HBV-infected hepatocytes are viable and express

167

168

169

170

171

172

173

174

175

176

177

no new viral antigens after mitosis

Finally, we determined the fate of individual HBV-infected cells that undergo mitosis; specifically, whether they undergo cell death or if they do indeed produce two uninfected daughter cells. To track living HBV-infected cells, we infected HepG2-NTCP cells with a reporter HBV that expresses turboRFP under the control of a TTR-promoter (rHBV-TTR-tRFP) (hereafter referred to as "tRFPHBV"). Given the long half-life of tRFP mRNA $\left(\sim 10 \mathrm{hrs}^{17}\right)$ and protein $\left(\sim 40\right.$ hours $\left.^{18}\right)$, we were able to track the mitosis of cells expressing cccDNA-encoded proteins and the daughter cells by live imaging.

HepG2-NTCP cells infected with tRFP-HBV were seeded in DMSO-free DMEM into chamber slides at $\sim 100,000$ cells per $\mathrm{cm}^{2}$ (about the concentration of a 1:1.5 split in comparison to previous experiments) allowing us to observe areas of high confluence (and therefore low mitosis) as well as low confluence/high mitosis. Live cell imaging was carried out over 36 hours. In areas of low confluence, we observed multiple instances of tRFP-positive cells undergoing mitosis, producing 2 living daughter cells containing tRFP. In high confluence areas, tRFP expression was stable in nondividing cells. Thus, division of HBV-infected cells does not induce cell death of daughter cells. 
183 We then tested if new tRFP was being expressed in the daughter cells by photo-bleaching all existing

184 tRFP. After exposure to 560/40nm light for 10 minutes, cells were allowed to recover for 5 hours and

185 reimaged. All tRFP-positive cells that had undergone mitosis in low confluence areas had lost

186 positive signals, whereas the cells that did not undergo mitosis (in high confluence areas and the

187 rare cells in low confluence areas) maintained positivity for tRFP. This suggests that HBV-infected

188 cells lose cccDNA directly after mitosis. 
In this study, we provide mathematical modelling and multiple lines of evidence unequivocally showing that cccDNA is not propagated to daughter cells after mitosis. Reduction of cccDNA levels in HepG2-NTCP (a hepatoma cell line) and HepaRG-NTCP (a hepatocyte-like cell line differentiated from bipotent progenitor cells) suggests that cccDNA is lost after mitosis of an infected cell. Thus, this non-cytolytic pathway for cccDNA clearance via cell mitosis is highly efficient.

Some reports have suggested partial maintenance of cccDNA molecules in daughter cells after mitosis ${ }^{4,10,13,14}$. These studies have generally used non-human model systems in which higher numbers of cccDNA per cell are generated: these include woodchuck ${ }^{4}$ and duck ${ }^{10}$ models, and HepAD38 cells ${ }^{13}$ in which viral replication and subsequent cccDNA formation is controlled by tetracycline. Moreover, nuclear import of mature nucleocapsids is a highly active feature in animal and over-expression models, but this route appears to contribute minimally to the cccDNA levels in human HBV infection models in vitro ${ }^{16}$. Future work is needed to confirm that these dynamics extend to the HBV-infected liver in humans.

Another difference to previous studies is the extent of mitosis induced: our experiments focused on only a few rounds of mitosis, whereas others performed splitting at higher dilutions and measured over longer periods - up to 30 weeks - after infection. For example, a previous report studied immune deficient UPA/SCID/beige mice implanted with HBV-infected hepatocytes, which repopulate the mouse liver after multiple rounds of mitosis ${ }^{8}$. In this system and consistent with the present data, rare non-dividing cells were found to maintain infection, while virus was eliminated in the bulk repopulated culture.

213 This data suggests heterogeneity in the host-cell population, where slow-growing or non-dividing 214 cells may effectively act as viral reservoirs (consistent with previously published mathematical 
models $\left.{ }^{19}\right)$. In agreement with this hypothesis, previous studies show non-random hepatocyte repopulation with selective clonal expansion being observed in people with chronic hepatitis $B$ and in animal models of hepatitis $\mathrm{B}^{20-24}$ and other liver injuries ${ }^{25}$. The effect of these heterogeneous cellular behaviours suggests that deciphering which route plays the major role for cccDNA persistence may identify a more efficient mechanism to clear cccDNA.

The results described here also impact the interpretation of future studies of cccDNA decay and therapeutic reduction. For example, our work suggests that a 2 -fold reduction in cccDNA per cell would result from a cumulative turnover of $\sim 35 \%$ of the cells in a culture. Thus, even slight levels of cytotoxicity or cell mitosis (particularly over long periods of culture) could dramatically reduce cccDNA levels. We therefore recommend that studies of investigational drugs or therapeutic approaches to specifically reduce cccDNA should determine carefully if the induction of mitosis contributes to the mechanism of action.

On the other hand, our work justifies a focus on therapeutic approaches that induce liver turnover to efficiently clear high levels of cccDNA. Potential strategies include immuno-modulators to induce liver turnover, though these would require considered application to avoid hepatic decompensation or carcinogenic risk. A targeted agent that induces the mitosis of specifically HBV-infected cells could overcome these risks. This theoretical agent would be a part of a combination of therapies to effect a cure: new infection events need to be inhibited to prevent de novo cccDNA formation, which can be achieved by concomitant treatment with viral entry inhibitors, capsid inhibitors, and/or nucleot(s)ide analogues.

In summary, we have uncovered a fundamental aspect of cccDNA dynamics that impacts the understanding of the viral persistence driving chronic HBV infections. Given the strong evidence that mitosis of infected hepatocytes results in uninfected daughter cells, greater focus should be applied 
241 to determine exactly how cccDNA can persist despite marked liver turnover during hepatic

242 inflammatory flares. The answer to this ongoing problem may hold the key to inducing a complete 243 cure of chronic hepatitis B. 
246 For wild-type HBV, virus stocks were purified from the supernatant of HepAD38 cells by heparin

247 affinity chromatography, as previously described ${ }^{26,27}$. For HBc-deficient HBV (and associated WT

248 control), virus was further concentrated using a 100-kDa Amicon Ultra-15 centrifugal filter

249 (UFC910024, Merck) from the supernatant of Huh7 cells transfected with plasmid constructs containing an over-length HBV genome (coding for HBV pgRNA and HBV proteins) and a complementing $\mathrm{HBc}$ overexpression plasmid (encoding $\mathrm{HBc}$ under a $\mathrm{CMV}$ promoter), as previously described ${ }^{16}$.

Reporter viruses rHBV-TTR-GLuc and rHBV-TTR-tRFP were generated in HepG2 cell lines that were stably co-transduced with a plasmid expressing HBV Polymerase, Surface Proteins and X and a recombinant HBV genome construct encoding HBV pgRNA with the HBsAg ORF replaced by an ORF encoding either turbo-RFP or Gaussia luciferase under a TTR promoter (as previously described ${ }^{28}$ ).

Reporter virus stocks were purified by heparin affinity chromatography and sucrose gradient ultracentrifugation of the supernatant of these cells, as previously described ${ }^{29}$.

Cell culture and HBV infection

HepaRG-NTCP (differentiated as previously described ${ }^{30}$ ), and HepG2-NTCP cells ${ }^{31}$ were used for in vitro infection. HepaRG-NTCP cells were cultivated in William's E media supplemented with $1.5 \%$ DMSO $^{32,33}$. HepG2-NTCP cells were maintained in DMSO-free Dulbecco's Modified Eagle's Medium ${ }^{31}$. HepG2-NTCP or dHepaRG-NTCP cells were seeded in 12-well plates and infected with HBV at up to $500 \mathrm{VGE} /$ cell in $250 \mu \mathrm{L}$ of culture media supplemented with $4 \% \mathrm{v} / \mathrm{v}$ polyethylene glycol 8000 (Sigma

267 Aldrich, St. Louis, MO USA) and 1.5\% v/v (for HepaRG-NTCP) or 2.5\% v/v DMSO (for HepG2-NTCP). 
9, and $12 \mathrm{dpi}$, cells were trypsinised and the cell suspension split in half; one half re-seeded in a new 12-well plate, and the other frozen at $-20^{\circ} \mathrm{C}$ for DNA extraction and cinqPCR analysis. In an alternate experimental setup (setup 2), instead of harvesting the second half of the cell suspension for DNA extraction, they were re-seeded in a new 12-well plate but did not undergo further splitting and all cells were harvested at $12 \mathrm{dpi}$.

\section{Detection of HBc-positive cells by immunofluorescence}

Cells were seeded onto $13 \mathrm{~mm}$ diameter glass coverslips in 24 -well plates. At cell harvest, culture media was aspirated from the cells, which were then washed with $1 \mathrm{~mL} 1$ xPBS and fixed with $300 \mu \mathrm{L}$ $4 \% \mathrm{w} / \mathrm{v}$ formaldehyde for 20 minutes at room temperature. Cells were permeabilised with $0.25 \% \mathrm{v} / \mathrm{v}$ Triton- $\mathrm{X}$ in $1 \mathrm{xPBS}$ for 20 minutes at room temperature. HBV core antigen was then detected using a 1:3000 dilution of polyclonal rabbit anti-HBc antibody (B0586, Dako, Denmark) ${ }^{33}$ overnight at $4^{\circ} \mathrm{C}$. After washing with 1xPBS, 1:500 AF545-conjugated goat anti-rabbit secondary antibody (A-11010, Invitrogen) and $2 \mu \mathrm{g} / \mathrm{mL}$ Hoechst 33342 (H1399, Invitrogen) in 1mL PBS was overlaid on the cells, which were incubated in the dark at room temperature for $1 \mathrm{hr}$. Fluorescence microscopy images were taken at 40x magnification with appropriate DAPI and Texas Red filter sets. For each sample, an area the size of 5 random fields of view were acquired by NIS Elements Advanced software (Nikon, Minato, Tokyo, Japan). Images were edited with ImageJ imaging software ${ }^{34}$ and quantified using llastik image classification, segmentation and analysis software (https://www.ilastik.org/, Version $1.3 .3)^{35}$

The cinqPCR protocol was carried out as previously described ${ }^{15,16,36}$. Total cellular DNA was extracted from harvested cells using a NucleoSpin ${ }^{\circledast}$ Tissue kit (740952, Macherey-Nagel, Düren, Germany) as per the manufacturer's instructions and eluted in $50 \mu \mathrm{L}$ of elution buffer. Ten $\mu \mathrm{L}$ of the DNA extract was digested in a $20 \mu \mathrm{L}$ restriction digestion reaction containing 1x CutSmart buffer (B7204, New 
England Biolabs, Ipswich, MA USA), 7.5U RecJ (M0264, NEB), and $10 \cup$ Hhal (R0139, NEB). The combined restriction digestion and exonuclease reaction was incubated for 4 rounds of a 15 min interval at $37^{\circ} \mathrm{C}$ followed by a $15 \mathrm{~min}$ interval at $42^{\circ} \mathrm{C}$. The enzymes were then heat-inactivated at $80^{\circ} \mathrm{C}$ for $20 \mathrm{~min}$. To circularise the digested fragments, a $10 \mu \mathrm{L}$ solution containing $500 \mathrm{U}$ of T4 DNA Ligase (M0202, NEB) in 1x CutSmart buffer and 3 mMol molecular-grade ATP (P0756, NEB) was added to the reaction. The reaction was incubated at $16^{\circ} \mathrm{C}$ for $2 \mathrm{hr}$, followed by an inactivation step of $80^{\circ} \mathrm{C}$ for $20 \mathrm{~min}$, and holding at $4^{\circ} \mathrm{C}$. A final linearization step was performed by adding $5 \mu \mathrm{L}$ solution containing $10 \cup \mathrm{Xbal}(\mathrm{R0145}, \mathrm{NEB})$ in $1 \times$ CutSmart buffer and incubating at $37^{\circ} \mathrm{C}$ for $2 \mathrm{hr}$, $80^{\circ} \mathrm{C}$ for $20 \mathrm{~min}$, and then storing at $4^{\circ} \mathrm{C}$ until further use.

For digital droplet (dd)PCR analysis, $2 \mu \mathrm{L}$ of the inverted product was put in a $20 \mu \mathrm{L}$ ddPCR reaction composed of 1x ddPCR Supermix for Probes (1863024, Biorad, Hercules, CA USA), 1x VIC-labelled TaqMan $^{\text {TM }}$ Copy Number Reference Assay for the human RNase P gene (4403328, Applied Biosystems, Foster City, CA USA), and 150 pMol of each HBV DNA-specific primer and probe (all synthesized by Eurofins Scientific, Luxembourg). The forward, reverse and probe sequences for the inverted HBV cccDNA fragment were 5'-CACTCTATGGAAGGCGGGTA-3', 5'-

ATAAGGGTCGATGTCCATGC-3', and 5'-FAM-AACACATAGCGCACCAGCA-BHQ1-3', respectively. The forward, reverse and probe sequences to detect total HBV DNA copies were 5'GTGTCTGCGGCGTTTTATCA-3', 5'- GACAAACGGGCAACATACCTT-3', and 5'-FAMTGAGGCATAGCAGCAGGATG-BHQ1-3', respectively. Droplets were generated according to the manufacturer's protocol using a QX200 Droplet Generator (Biorad). Intra-droplet PCR was carried out using the following protocol: an initial 10min denaturation, enzyme activation and droplet stabilization step at $95^{\circ} \mathrm{C}$; followed by 40 cycles of a 10 s denaturation step at $95^{\circ} \mathrm{C}$, a 15 s annealing step at $54^{\circ} \mathrm{C}$ and a 20 s elongation step at $68^{\circ} \mathrm{C}$, finished with a 10 minute enzyme deactivation step at $95^{\circ} \mathrm{C}$. Products were then stored at $12^{\circ} \mathrm{C}$ until droplet reading using a QX200 Droplet Reader (Biorad), quantification using FAM and VIC channels, and data analysis using QuantaSoft (Biorad). 
HepG2-NTCP cells infected with $~ 500$ VGE/cell of rHBV-TTR-GLuc in 24-well plates and serially split

HepG2-NTCP cells infected as described above with 500 VGE/cell of tRFP-HBV in a 24-well plate. At 3 days post-infection, cells were seeded into Nunc Lab-Tek ${ }^{\mathrm{TM}}$ 8-well chamber (ThermoFisher Scientific, 177402PK) slides at a concentration of $\sim 100,000$ cells per $\mathrm{cm}^{2}$ in $400 \mu \mathrm{L}$ DMSO-free DMEM to stimulate cell mitosis. After allowing cells to attach for 8 hours post-seeding, sliders were transferred into a humidity-, heat- and $\mathrm{CO}_{2}$-controlled chamber connected to a Zeiss AxioVert $200 \mathrm{M}$ After a recovery period of 4 hours, images from the same fields of view were then acquired exactly as described above with identical settings. Images were acquired by Zeiss Zen Pro imaging software 343 (Carl Zeiss Microscopy $\mathrm{GmbH}$ ) and edited with ImageJ imaging software ${ }^{34}$. 
346 This work received funding from: the German Centre for Infection Research (DZIF) TTU Hepatitis

347 Projects 5.807 and 5.704 (T.T. and S.U.); the Deutsche Forschungsgemeinschaft (DFG, German

348 Research Foundation) - Project number 240245660; SFB 1129 (B.Z. and S.U.) and - Project number

349272983813 - TRR179 (TP 15) (S.U.); the Australian Centre for HIV and Hepatitis Virology Research

350 (T.T.); and the Australian National Health and Medical Research Council Ideas Grant APP2002565

351 (T.T. and M.D.), Program Grants APP1053206, APP1149976 and Project grants APP1107178 and

352 APP1108422 (J.G.); and the Robert W. Storr Bequest to the Sydney Medical Foundation (J.G.).

353 Microscopy was performed at the Westmead Scientific Platforms, which are supported by the

354 Westmead Research Hub, the Cancer Institute New South Wales, the National Health and Medical

355 Research Council, and the lan Potter Foundation.

356

357

Acknowledgements

358

We acknowledge: Drs. Yi Ni and Florian A. Lempp for reagents (cell lines and HBV inoculum); and Lisa

359

Walter, Anja Rippert, Franziska Schlund, Dr. Christa Kuhn, and Westmead Research Hub for their

360

technical assistance. We are grateful to Dr. Zhenfeng Zhang for helpful discussions, Miriam Kleinig

361 for proofreading and Prof. Dr. Ralf Bartenschlager for continuous support.

362

363

Data availability statement

364 All data generated or analysed during this study are included in this published article (and its 365 supplementary information files).

366 
368 Stephan Urban is co-applicant and co-inventor on patents protecting HBV preS-derived lipopeptides

369 (Myrcludex B) for the use of HBV/HDV entry inhibitors. Ulrike Protzer a co-founder and shareholder

370 of SCG Cell Therapy. The other authors in this study declare no relevant competing interests.

\section{Author contributions}

373 T.T. conceived the concept of the project, designed and carried out the experiments, analyzed the

374 data, generated figures and wrote the manuscript; B.Z. designed and carried out the HBc-deficient

375 mutant experiments and assisted in writing the manuscript; H.Z., S.C., and V.H. assisted in the

376 experimental design of, carried out experiments, and assisted in writing the manuscript; J.W.

377 generated the reporter viruses, assisted in experimental design and contributed to writing the

378 manuscript; U.P. provided funding for J.W. and assisted in writing the manuscript; M.D. and J.G.

379 provided funding for T.T., V.H., and parts of the project, and contributed to writing the manuscript;

380 S.U. provided funding for T.T. and B.Z. and parts of the project, contributed intellectual input on

381 experimental design, and contributed to writing the manuscript. 
Stanaway, J. D. et al. The global burden of viral hepatitis from 1990 to 2013: findings from the Global Burden of Disease Study 2013. Lancet 388, 1081-1088, doi:10.1016/S01406736(16)30579-7 (2016).

2 Tu, T., Block, J. M., Wang, S., Cohen, C. \& Douglas, M. W. The lived experience of chronic hepatitis B: a broader view of its impacts and why we need a cure. Viruses 12, 515 (2020).

3 Moraleda, G. et al. Lack of effect of antiviral therapy in nondividing hepatocyte cultures on the closed circular DNA of woodchuck hepatitis virus. J Virol 71, 9392-9399 (1997).

4 Dandri, M., Burda, M. R., Will, H. \& Petersen, J. Increased hepatocyte turnover and inhibition of woodchuck hepatitis $B$ virus replication by adefovir in vitro do not lead to reduction of the closed circular DNA. Hepatology 32, 139-146, doi:10.1053/jhep.2000.8701 (2000).

5 Lucifora, J. et al. Specific and nonhepatotoxic degradation of nuclear hepatitis B virus cccDNA. Science 343, 1221-1228, doi:10.1126/science.1243462 (2014).

6 Guidotti, L. G. et al. Viral clearance without destruction of infected cells during acute HBV infection. Science 284, 825-829 (1999).

7 Murray, J. M., Wieland, S. F., Purcell, R. H. \& Chisari, F. V. Dynamics of hepatitis B virus clearance in chimpanzees. Proc Natl Acad Sci U S A 102, 17780-17785 (2005).

8 Allweiss, L. et al. Proliferation of primary human hepatocytes and prevention of hepatitis B virus reinfection efficiently deplete nuclear cccDNA in vivo. Gut 67, 542-552, doi:10.1136/gutjnl-2016-312162 (2018).

9 Lok, A. S., Zoulim, F., Dusheiko, G. \& Ghany, M. G. Hepatitis B cure: From discovery to regulatory approval. J Hepatol 67, 847-861, doi:10.1016/j.jhep.2017.05.008 (2017).

10 Reaiche-Miller, G. Y. et al. Duck hepatitis B virus covalently closed circular DNA appears to survive hepatocyte mitosis in the growing liver. Virology 446, 357-364, doi:10.1016/j.virol.2013.08.014 (2013).

11 Murray, J. M. \& Goyal, A. In silico single cell dynamics of hepatitis B virus infection and clearance. J Theor Biol 366, 91-102, doi:10.1016/j.jtbi.2014.11.020 (2015).

12 Lutgehetmann, M. et al. In vivo proliferation of hepadnavirus-infected hepatocytes induces loss of covalently closed circular DNA in mice. Hepatology 52, 16-24, doi:10.1002/hep.23611 (2010).

13 Li, M., Sohn, J. A. \& Seeger, C. Distribution of Hepatitis B Virus Nuclear DNA. J Virol 92, doi:10.1128/JVI.01391-17 (2018).

14 Zhu, Y. et al. Kinetics of hepadnavirus loss from the liver during inhibition of viral DNA synthesis. J Virol 75, 311-322 (2001).

$15 \mathrm{Tu}, \mathrm{T}$. et al. A novel method to precisely quantify Hepatitis B Virus covalently closed circular (ccc)DNA formation and maintenance. Antiviral Res, 104865, doi:10.1016/j.antiviral.2020.104865 (2020).

16 Tu, T., Zehnder, B., Qu, B. \& Urban, S. De novo synthesis of Hepatitis B virus nucleocapsids is dispensable for the maintenance and transcriptional regulation of cccDNA JHEP Reports, doi:10.1016/j.jhepr.2020.100195 (2020).

17 Yang, E. et al. Decay rates of human mRNAs: correlation with functional characteristics and sequence attributes. Genome Res 13, 1863-1872, doi:10.1101/gr.1272403 (2003).

18 Shaner, N. C. et al. Improving the photostability of bright monomeric orange and red fluorescent proteins. Nat Methods 5, 545-551, doi:10.1038/nmeth.1209 (2008).

19 Lythgoe, K. A., Lumley, S. F., Pellis, L., McKeating, J. A. \& Matthews, P. C. Estimating hepatitis $B$ virus cccDNA persistence in chronic infection. Virus Evol 7, veaa063, doi:10.1093/ve/veaa063 (2021).

20 Mason, W. S. et al. HBV DNA Integration and Clonal Hepatocyte Expansion in Chronic Hepatitis B Patients Considered Immune Tolerant. Gastroenterology 151, 986-998 e984, doi:10.1053/j.gastro.2016.07.012 (2016). 
21 Mason, W. S., Jilbert, A. R. \& Summers, J. Clonal expansion of hepatocytes during chronic woodchuck hepatitis virus infection. Proc Natl Acad Sci U S A 102, 1139-1144, doi:0409332102 [pii] 10.1073/pnas.0409332102 (2005).

22 Mason, W. S., Liu, C., Aldrich, C. E., Litwin, S. \& Yeh, M. M. Clonal expansion of normalappearing human hepatocytes during chronic hepatitis B virus infection. J Viro/ 84, 83088315, doi:10.1128/JVI.00833-10 (2010).

23 Mason, W. S. et al. Detection of clonally expanded hepatocytes in chimpanzees with chronic hepatitis B virus infection. J Virol 83, 8396-8408, doi:JVI.00700-09 [pii] 10.1128/JVI.00700-09 (2009).

$24 \mathrm{Tu}, \mathrm{T}$. et al. Clonal expansion of hepatocytes with a selective advantage occurs during all stages of chronic hepatitis B virus infection. J Viral Hepat 22, 737-753, doi:10.1111/jvh.12380 (2015).

25 Wei, Y. et al. Liver homeostasis is maintained by midlobular zone 2 hepatocytes. Science 371, doi:10.1126/science.abb1625 (2021).

26 Ladner, S. K. et al. Inducible expression of human hepatitis B virus (HBV) in stably transfected hepatoblastoma cells: a novel system for screening potential inhibitors of HBV replication. Antimicrob Agents Chemother 41, 1715-1720, doi:10.1128/AAC.41.8.1715 (1997).

27 Lempp, F. A. et al. Evidence that hepatitis B virus replication in mouse cells is limited by the lack of a host cell dependency factor. J Hepatol 64, 556-564, doi:10.1016/j.jhep.2015.10.030 (2016).

28 Wing, P. A. et al. A dual role for SAMHD1 in regulating HBV cccDNA and RT-dependent particle genesis. Life Sci Alliance 2, doi:10.26508/Isa.201900355 (2019).

29 Wettengel, J. M. et al. Rapid and Robust Continuous Purification of High-Titer Hepatitis B Virus for In Vitro and In Vivo Applications. Viruses 13, doi:10.3390/v13081503 (2021).

30 Gripon, P. et al. Infection of a human hepatoma cell line by hepatitis B virus. Proc Natl Acad Sci U S A 99, 15655-15660, doi:10.1073/pnas.232137699 (2002).

$31 \mathrm{Ni}, \mathrm{Y}$. et al. Hepatitis B and D viruses exploit sodium taurocholate co-transporting polypeptide for species-specific entry into hepatocytes. Gastroenterology 146, 1070-1083, doi:10.1053/j.gastro.2013.12.024 (2014).

32 Gripon, P. et al. Infection of a human hepatoma cell line by hepatitis B virus. Proc Natl Acad Sci U S A 99, 15655-15660, doi:10.1073/pnas.232137699 (2002).

33 Schulze, A., Mills, K., Weiss, T. S. \& Urban, S. Hepatocyte polarization is essential for the productive entry of the hepatitis B virus. Hepatology 55, 373-383, doi:10.1002/hep.24707 (2012).

34 Schneider, C. A., Rasband, W. S. \& Eliceiri, K. W. NIH Image to ImageJ: 25 years of image analysis. Nat Methods 9, 671-675 (2012).

35 Berg, S. et al. ilastik: interactive machine learning for (bio)image analysis. Nat Methods 16, 1226-1232, doi:10.1038/s41592-019-0582-9 (2019).

36 Zehnder, B., Urban, S. \& Tu, T. A Sensitive and Specific PCR-based Assay to Quantify Hepatitis B Virus Covalently Closed Circular (ccc) DNA while Preserving Cellular DNA. Bio-protocol 11, e3986, doi:10.21769/BioProtoc.3986 (2021). 
Figure 1

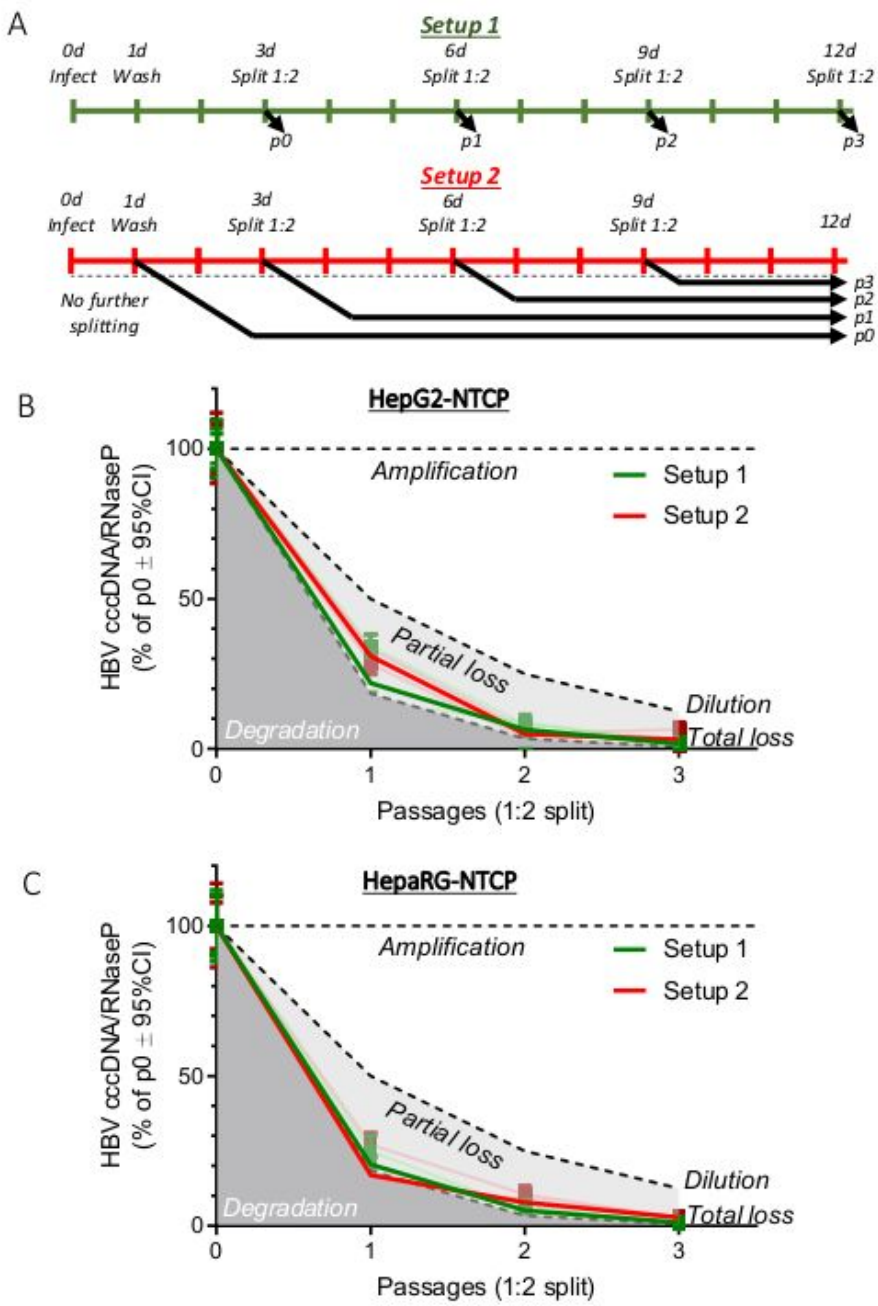

Figure 1. cccDNA levels are profoundly reduced after rounds of cell mitosis in HepG2-NTCP and HepaRGNTCP cells.

(A) Two experimental setups were used to study the fate of cccDNA following cell mitosis with the main difference being whether cells left over from the splitting were immediately lysed (Setup 1, green) or cultured until 12dpi before DNA was extracted and analysed by cinqPCR (Setup 2, red).

(B and C) Dilution and complete loss models (top and bottom dashed lines, respectively) predict different levels of cccDNA decrease ( 50 and $18.4 \%$ of the pre-split value per passage, respectively). The area between these two models (light grey) represents a model where there is partial loss of cccDNA during mitosis. Levels higher than the dilution model would suggest that cccDNA is somehow amplified following mitosis (amplification, white area), whereas levels lower than the loss model (degradation, dark grey area) would suggest other cccDNA degradation mechanisms, e.g. cytokine-induced cccDNA degradation. HepG2-NTCP (top) and HepaRG-NTCP (bottom) cells were infected and harvested as per setup 1 or 2 . The cccDNA decrease according to the dilution and loss models (dashed lines) were predicted using only the 0 passage $(\mathrm{p} 0)$ timepoint. Data points (connected by light lines) represent 3 independent experiments and the mean observed values for setup 1 or setup 2 are shown in solid green and red lines, respectively.

\section{Figure 1}


Figure 2

A

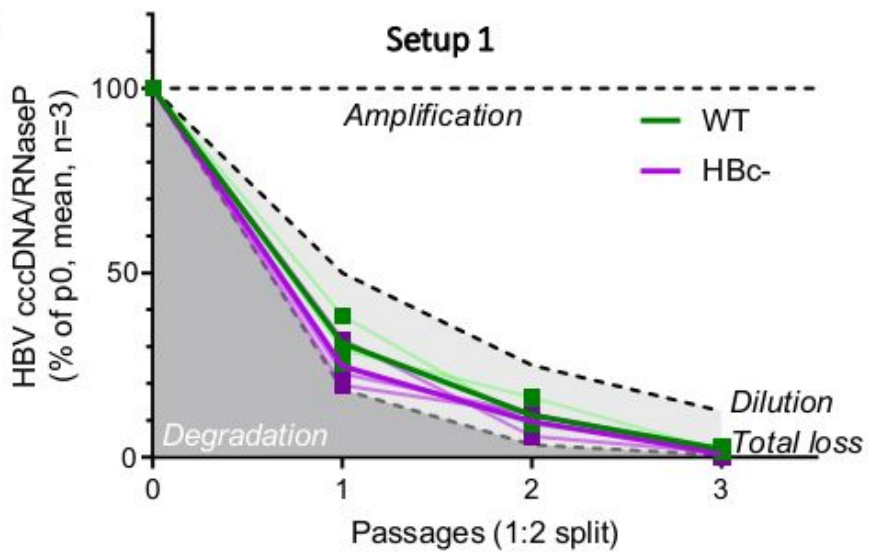

B

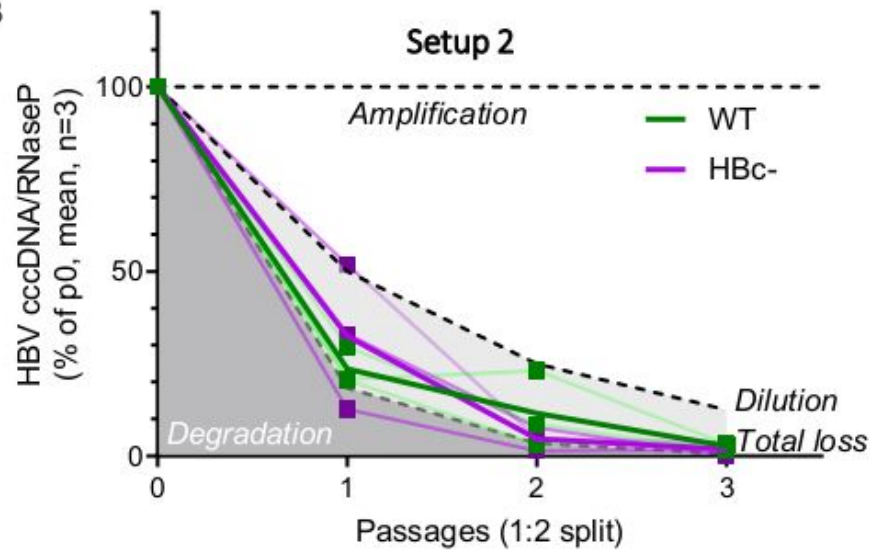

Figure 2. Mitosis-associated cccDNA loss is unaffected by de novo viral replication.

HepG2-NTCP cells were infected with either wild-type HBV (green) or replication-deficient HBV mutants containing a stop-codon in the $\mathrm{HBc}$ ORF (purple). The infected cells were then serially split according to Setup 1 (A) or Setup 2 (B), as described in Figure 1A. Data points (connected by light lines) represent 3 independent experiments and the mean observed values are shown in solid green and purple lines for WT and HBCdeficient mutant-infected cells, respectively.

\section{Figure 2}

See image above for figure legend

Figure 3 
Figure 4

A
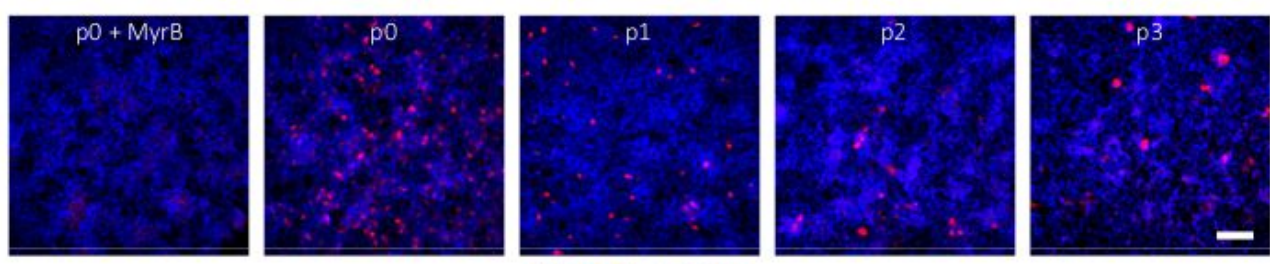

Hoechst $\mathrm{HBC}$

B

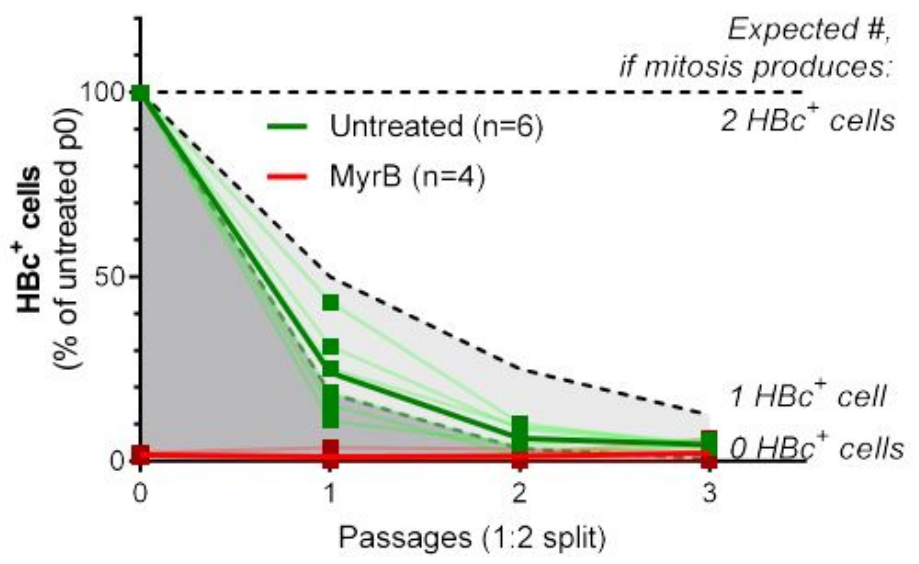

Figure 4. Number of $\mathrm{HBc}$-positive cells decrease with serial passaging consistent with cccDNA loss with mitosis.

(A) HBV-infected HepG2-NTCP cells were treated as per setup 2 (Figure 1A), fixed at 12 days postinfection, and visualised by fluorescence microscopy ( $\mathrm{HBC}$ in red, Hoechst staining for cell nuclei in blue). Scale bar $=100 \mu \mathrm{m}$. MyrB = cells pre-treated with HBV entry inhibitor Myrcludex B as a negative control. (B) A marked decrease in HBc-positive cells was observed with each additional split (green), highly consistent with the loss of HBV cccDNA in daughter cells of infected hepatocytes. Dashed lines represent the number of cells expected per split if 1 or 2 daughter cells (dilution model) or 0 daughter cells (complete loss model) were $\mathrm{HBc}$-positive following mitosis. The percentage of $\mathrm{HBc}$-positive cells was calculated by dividing the number of $\mathrm{HBc}$-positive cells by the number of nuclei in 5 randomlypicked fields of view. Few RFP-positive cells were observed in those pre-treated with the HBV entry inhibitor Myrcludex B (red), showing bona fide infection had occurred.

\section{Figure 4}


Figure 5
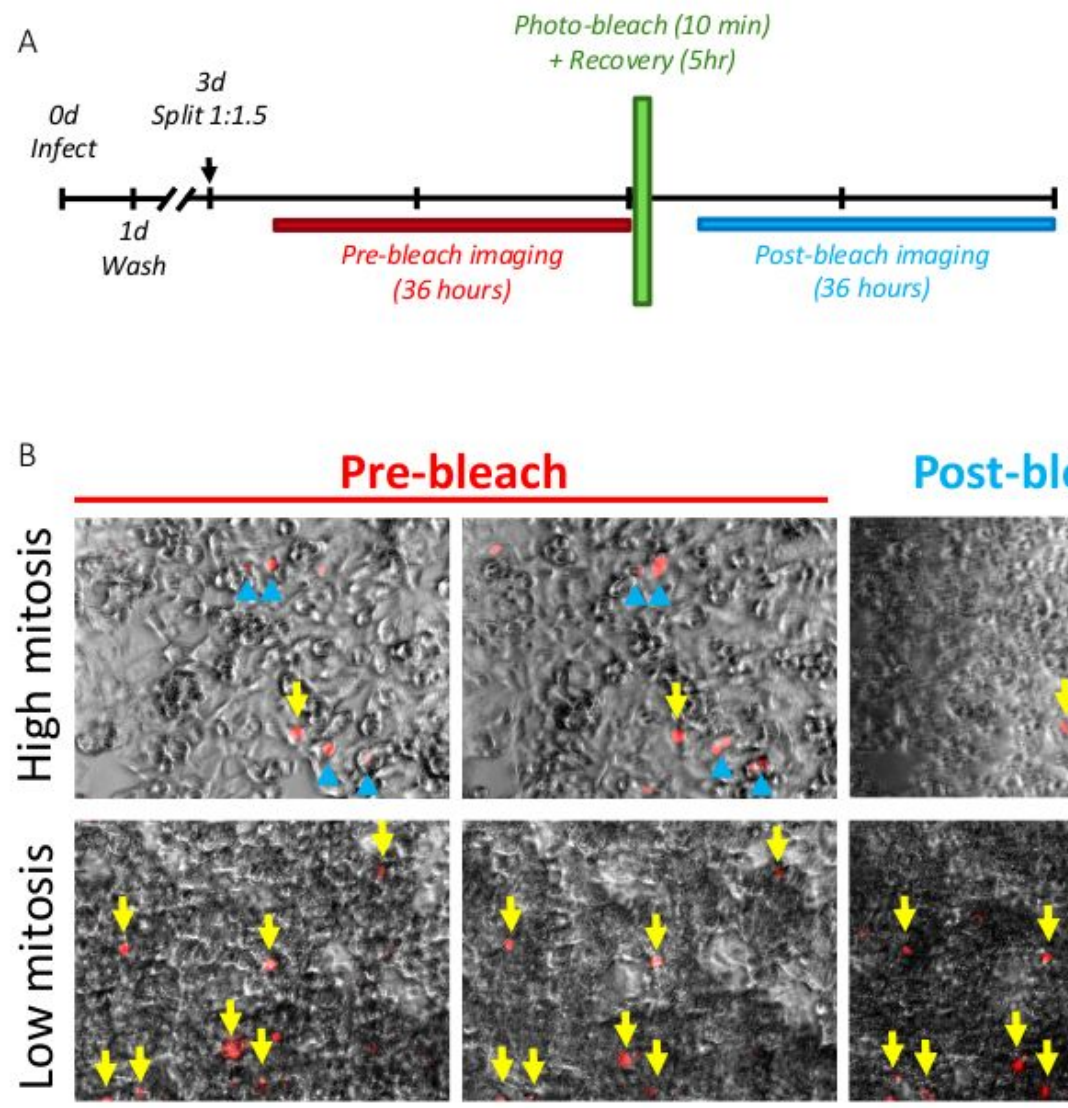

\section{Post-bleach}
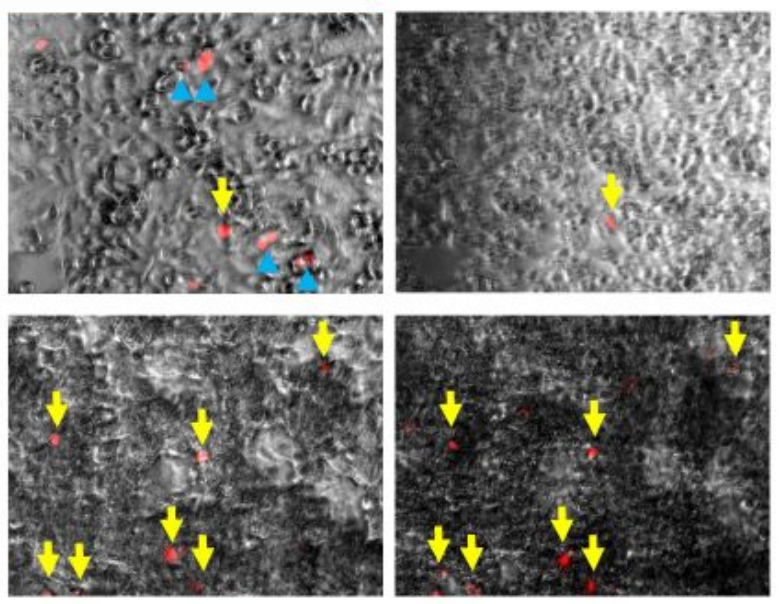

Figure 5. Live-cell imaging shows that HBV-infected cells that undergo mitosis produce viable daughter cells free of transcriptionally active cccDNA.

(A) HepG2-NTCP cells infected with a tRFP-expressing reporter HBV and reseeded at a lower density (equivalent to a 1:1.5 split) in a chamber-slide. After allowed to reattach to the slide bottom for 12 hours, the cells were live-imaged in a humidity-, heat- and $\mathrm{CO}_{2}$-controlled chamber ( 1 capture every 15 minutes for 36 hours). Cells were then photo-bleached with 10 minutes exposure of 560/40nm laser light and cells were allowed to recover for 5 hours to limit excess photo-toxicity associated with image acquisition. Live acquisition was then continued for another 36 hours in the post-bleaching stage. Acquisition was measured at $40 \mathrm{x}$ with excitation filters at $560 / 40 \mathrm{~nm}$ and emission filters at $630 / 75 \mathrm{~nm}$ for RFP fluorescence and white light for phase contrast.

(B) Stills from live imaging in pre-bleach (left and centre panels) and post-bleach (right panel) time-points in fields of view with low confluence (high mitosis, top row) or high confluence (low mitosis, bottom row). RFP-positive cells that did not undergo mitosis in the pre-bleach period are marked with a yellow arrow, while cells in which mitosis was observed are marked with a blue triangle. No RFP-positive cells that underwent mitosis maintained positive staining after bleaching.

\section{Figure 5}

\section{Supplementary Files}

This is a list of supplementary files associated with this preprint. Click to download. 
- figureS1.jpg 